\title{
Risk factors for invasive pulmonary fungal infection in patients with hematological malignancies not receiving hematopoietic stem cell transplant
}

\author{
R. HU⿱1${ }^{1}$ X. Y. JIANG ${ }^{1, *}, \mathrm{Y}^{2, *}$ \\ ${ }^{1}$ Nursing School of Fujian Medical University. 1 Xueyuan Road, Shangjie Town, Minhou County, Fuzhou, Fujian 350108, P.R. China; ${ }^{2}$ Union \\ Hospital Affiliated with Fujian Medical University. 29 Xinquan Road, Fuzhou 350001, Fujian, P.R. China
}

*Correspondence: jiangxy320@126.com,wuyong9195@126.com

Received April 2, 2012 / Accepted May 30, 2012

\begin{abstract}
We investigated the incidence, pathogens and risk factors of invasive pulmonary fungal infection (IPFI) in patients with hematological malignancies who did not receive hematopoietic stem cell transplantation (HSCT). Of the 323 patients included, 106 had IPFI with confirmed diagnosis or clinical diagnosis, 111 had pulmonary bacterial infections, and 106 did not have pulmonary infections. The risk factors for IPFI were explored through logistic univariate and multivariate analysis. The incidence of IPFI in patients with hematological malignancies but without HSCT was 3.5\%. The leading pathogen was Candida albicans which accounted for $50.7 \%$ of the infections, and the second one was Aspergillus which accounted for $37.3 \%$ of the infections. The main risk factors for these patients were days of hospitalization, history of IPFI, agranulocytosis, concomitant hypoproteinemia, number of antibiotics being used, concomitant bacterial sepsis, and age. Furthermore, Nystatin mouthwash was protective against IPFI. Among patients with hematological malignancies, IPFI causes the highest proportion of deaths. We have identified two important pathogens and several risk factors as well as one factor protective against IPFI. Awareness of risk factors and reduction of pathogens can decrease the incidence of IPFI.
\end{abstract}

Key words: fungemia, mycoses/prevention and control, epidemiology, Nystatin, hematologic neoplasms/complications

Invasive pulmonary fungal infections (IPFI) have high morbidity, high mortality, and a low detection rate in laboratories and in clinical practice. Though patients with IPFI deteriorate rapidly, clinical manifestations are usually nonspecific and frequently masked by underlying diseases. This leads to misdiagnosis of IPFI and delayed treatment. Together, these problems, along with the influence of underlying diseases, significantly increase mortality. When IPFI patients do not receive timely treatment, their mortality is as high as $30 \%$ to $80 \%$ [1].

Risk factors for IPFI include long-term broad-spectrum antibiotic use, long-term glucocorticoid use, agranulocytosis, invasive procedures, long-term stay in intensive care unit, hematopoietic stem cell transplantation (HSCT), total parenteral nutrition, concomitant hypoproteinemia, diabetes, chronic obstructive pulmonary disease, cancer, and AIDS [1-7]. However, risk factors for IPFI are seldom investigated for patients with hematological malignancies, and most investigations that do occur were focused on patients receiving HSCT [8-10]. Some patients with hematological malignancies who have not received HSCT still develop IPFI, even they were not identified as having any of the risk factors. Therefore, it is imperative to investigate the risk factors for IPFI in these patients. The present study examines the overall incidence of IPFI and the pathogens involved in patients with hematological malignancies who did not receive HSCT. Controls were patients from the same group who developed pulmonary bacterial infection and patients without pulmonary infection. We identify risk factors and a protective factor for IPFI. Our results may provide evidence and direction to prevent and treat IPFI.

\section{Patients and methods}

Patients. Patients were selected from the Electronic Medical Record Management System and from written medical records of the Affiliated Union Hospital of Fujian Medical University. The institutional review board of the Affiliated Union Hospital of Fujian Medical University approved this retrospective review and study. The patients who were included were (1) hospitalized in the Department of Hematology at our 
hospital between January 1, 2008 and December 31, 2009 and (2) diagnosed with hematological malignancies that included leukemia, lymphoma, multiple myeloma, and myelodysplastic syndrome. Patients who received HSCT were excluded. The medical records of the included patients were carefully reviewed to collect all required information for this study. The causes of IPFI were detected by culture of blood, sputum, or throat swab, or by a galactomannan test.

\section{Diagnosis of IPFI according to Deng 2006}

Definition. Our definition of IPFI does not include bronchial and pulmonary fungal infections attributed to fungal parasitism and sensitivity. We classified IPFI as primary and secondary IPFI. The common fungi causing IPFI include Candida, Aspergillus, Cryptococcus, Zygomycetes (Mucor), Pneumocystis, etc. The diagnosis of IPFI is based on the host factors, clinical characteristics, findings in microbiological examination, and histopathological examination. The host factors need to be considered in the clinical diagnosis of IPFI where as the pulmonary infection due to other pathogens and non-infection disease should be excluded. The diagnoses of IPFI can be classified as confirmed diagnosis, clinical diagnosis, and suspected diagnosis.

Confirmed diagnosis of IPFI. A diagnosis of IPFI is considered confirmed when the IPFI has at least one host factor, one major characteristic or two minor characteristics of pulmonary infection and one finding in microbiological or histopathological examination.

1. Mold: Lung tissues were processed for histological or cytochemical examination to detect the mycelium or spherical bodies (filamentous fungi) and corresponding lung lesion. Lung tissues, chest fluid, or blood was positive for mold in fungal culture. When the blood was positive for Aspergillus or Penicillium (except for Penicillium marneffei), consider the clinical findings in order to exclude contamination.
2. Yeast: Lung tissues were processed for histological or cytochemical examination to detect yeasts or pseudohyphae. Lung tissues, chest fluid, or blood was positive for yeasts or microscopy if it had Cryptococcus.

3. Pneumocystis: The cystica, trophozoite, or intracystic bodies of pneumocystis were found in the lung tissues, Bronchoalveolar Lavage Fluid (BALF), and sputum.

Clinical diagnosis of IPFI. A clinical diagnosis of IPFI required at least one host factor, one major characteristic or two minor characteristics of pulmonary infection, and one finding by microbiological or histopathological examination.

Suspected diagnosis of IPFI. A suspected diagnosis of IPFI required at least one host factor and one major characteristic or two minor characteristics of pulmonary infection.

The criteria for the each diagnosis of IPFI are presented in Table 1.

Statistical analysis. The demographics, characteristics, complications, and medications of the included patients were summarized as $\mathrm{n}(\%)$ and compared among groups with the Pearson Chi-square test or, if cell numbers were less than five, with the Fisher's exact test. The IPFI prevalence rate was calculated as the total IPFI frequency divided by total hospitalization. Pathogens that caused IPFI were summarized in a frequency table. A step-wise logistic regression identified the association of IPFI with multiple risk factors by comparisons of the IPFI group with the pulmonary bacterial infection group and with the non-pulmonary infection group. Variables from simple logistic regression analysis were selected based on a significance level of 0.2 and were used in multiple logistic regression analysis with a forward selection (Method: LR). All statistical assessments were two-tailed and considered significant when $P<0.05$. An adjusted significance level $0.0167(0.05 / 3)$ was used for post-hoc pair-wise comparisons. Statistics were analyzed with SPSS 15.0 statistics software (SPSS Inc, Chicago, IL, USA).

Table 1. Criteria for the diagnosis of IPFI

\begin{tabular}{lcccc}
\hline & Host factor & Clinical characteristics & Microbiological examination & Pathological examination \\
\hline Confirmed diagnosis & $+^{*}$ & + & $+^{\Delta}$ & + \\
Clinical diagnosis & + & + & + & - \\
preliminary diagnosis & + & + & - & - \\
\hline
\end{tabular}

Notes: ${ }^{*}$ primary IPFI does not need to include host factor; ${ }^{\Delta}$ indicates positive microbiological culture for lung tissue, chest fluid, and blood (except for Pneumocystis).

Host factors (1) neutropenia is present (neutrophil count is $<0.5 \times 109 / \mathrm{L}$ ) for $>10 \mathrm{~d}$; (2) Body temperature was $>38^{\circ} \mathrm{C}$ or $<36^{\circ} \mathrm{C}$ with one of following findings: (a) sustained neutropenia (>10 days) was found in past 60 days; (b) patients were treated with immunosuppressants in past 30 days or were undergoing treatment with immunosuppressants; (c) patients had a history of IPFI; (d) patients had HIV infection; (e) patients had symptoms and signs of graft versus host diseases; (f) patients were treated with steroids for more than 3 weeks; or (g) patients had underlying chronicle diseases, trauma, surgery, long ICU stay after surgery, long term mechanical ventilation, indwelling catheter, total parenteral nutrition, or long-term treatment with broad spectrum antibiotic, etc.

Clinical characteristics: Characteristics of invasive pulmonary Aspergillosis in chest X ray and chest CT included subpleural nodule-like markings found at an early stage, and halo sign found several days later; $10-15$ days later, liquefaction and necrosis were found and cavity markings or crescent sign were present. Characteristics of Pneumocystis pneumonia in chest CT included: ground-glass-like changes in pulmonary interstitium and the presence of hypoxemia. Minor characteristics were: (a) symptoms and signs of pulmonary infection; (b) pulmonary infusion markings in imaging; and (c) sustained pyrexia for 96 hours that was nonresponsive to aggressive antimicrobial therapy. 


\section{Results}

We identified 1858 patients with 6047 hospitalizations, and among these patients, 323 cases of IPFI or pulmonary infection were found (Table 1). The medical records of the identified patients were retrospectively reviewed, and the patients were divided into three groups:

(1)The IPFI group included those diagnosed with IPFI based on the criteria for the Diagnosis and Treatment of IPFI in Patients with Hematological Malignancies [11] as described in patients and methods. These 212 patients included one confirmed diagnosis, 105 clinical diagnoses, and 106 preliminary diagnoses. Of these IPFI patients, the study included the 106 patients with either a confirmed or clinical diagnosis, with a mean age of $47.4 \pm 19.3$ years (Table 2). These patients had a higher incidence of hypoalbuminemia and electrolyte disorder, but a lower incidence of gastroin- testinal and oral fungal infections than the other groups of patients (Table 3 ). These patients were treated at higher rates than the other two groups of patients with vein nutrition infusion, albumin infusion, central vein construction, more than one type of immunosuppressant, more than one type of antibiotic, antibiotic for more than one day, and Nystatin for less than eight days. The IPFI prevalence rate was $3.82 \%$ in 2008 and $3.23 \%$ in 2009 (Table 4).

(2)The pulmonary bacterial infection group, which was the first control group, included 111 patients diagnosed with pulmonary bacterial infection with a mean age of $40.9 \pm$ 18.1 years (Table 2 ).

(3)The non-pulmonary infection group, which was the second control group, included 106 patients randomly selected of those who did not develop a pulmonary infection during their hospitalization with a mean age of $40.3 \pm 16.2$ years (Table 2).

Table 2. Demographic information and characteristics of participants by group.

\begin{tabular}{|c|c|c|c|c|}
\hline & IPFI $(n=106)$ & Pulmonary bacterial infection $(\mathrm{n}=111)$ & Non-pulmonary infection $(\mathrm{n}=106)$ & $P$-value \\
\hline \multicolumn{5}{|l|}{ Age } \\
\hline$<60 \mathrm{yrs}$ & $69(65.1)$ & $92(82.9)$ & $89(84.0)$ & $0.001^{* \ddagger}$ \\
\hline$\geqq 60$ yrs & $37(34.9)$ & $19(17.1)$ & $17(16.0)$ & \\
\hline \multicolumn{5}{|l|}{ Sex } \\
\hline Males & $65(61.3)$ & $61(55.0)$ & $65(61.3)$ & 0.543 \\
\hline Females & $41(38.7)$ & $50(45.0)$ & $41(38.7)$ & \\
\hline Previous IPFI history & $21(19.8)$ & $5(4.5)$ & $8(7.5)$ & $0.001^{* \neq \neq}$ \\
\hline Hospital days & $34.9 \pm 21.1$ & $23.5 \pm 14.7$ & $11.2 \pm 11.6$ & $<0.001^{* \neq \neq}$ \\
\hline$<8$ days & $5(4.7)$ & $19(17.1)$ & $60(56.6)$ & $<0.001^{*}+*$ \\
\hline 9 to 30 days & $47(44.3)$ & $67(60.4)$ & $42(39.6)$ & \\
\hline$>30$ days & $54(50.9)$ & $25(22.5)$ & $4(3.8)$ & \\
\hline Disease diagnosis & & & & $0.031^{*}$ \\
\hline $\mathrm{AL}$ & $78(73.6)$ & $86(77.5)$ & $69(65.1)$ & \\
\hline CL & $4(3.8)$ & $3(2.7)$ & $4(3.8)$ & \\
\hline NHL & $15(14.2)$ & $15(13.5)$ & $31(29.2)$ & \\
\hline MM & $6(5.7)$ & $6(5.4)$ & $1(0.9)$ & \\
\hline MDS & $3(2.8)$ & $1(0.9)$ & $1(0.9)$ & \\
\hline Disease status & & & & $<0.001^{* \neq}$ \\
\hline Complete remission & $21(19.8)$ & $23(20.7)$ & $56(52.8)$ & \\
\hline Initial diagnosis & $58(54.7)$ & $65(58.6)$ & $31(29.2)$ & \\
\hline Recurrence & $27(25.5)$ & $23(20.7)$ & $19(17.9)$ & \\
\hline \multicolumn{5}{|l|}{ Medical history } \\
\hline Diabetes mellitus & $16(15.1)$ & $7(6.3)$ & $5(4.7)$ & $0.014^{*}$ \\
\hline Hypertension & $13(12.3)$ & $4(3.6)$ & $5(4.7)$ & $0.037^{*}$ \\
\hline Coronary artery disease & $6(5.7)$ & $3(2.7)$ & $1(0.9)$ & 0.147 \\
\hline Hyperlipidemia & $2(1.9)$ & $1(0.9)$ & $15(14.2)$ & $<0.001^{*}$ \\
\hline COPD & $0(0)$ & $1(0.9)$ & $2(1.9)$ & NA \\
\hline Liver disease & $7(6.6)$ & $3(2.7)$ & $4(3.8)$ & 0.383 \\
\hline
\end{tabular}

Abbreviations: IPFI, invasive pulmonary fungal infections; AL, acute leukemia; CL, chronic leukemia; NA, not assessed because the cell number was zero; NHL, non-Hodgkin lymphoma; MM, multiple myeloma; MDS, myelodysplastic syndrome; COPD, chronic obstructive pulmonary disease.

Data are summarized as $\mathrm{n}(\%)$ and compared among groups by the Pearson Chi-square test or, if cell numbers were $<5$, by the Fisher's exact test.

" Indicates a significant difference among the three groups with $P<0.05$.

$\dagger$ Indicates a significant difference between IPFI and Pulmonary bacterial infection with $P<0.0167(0.05 / 3)$.

$\ddagger$ Indicates a significant difference between IPFI and non-Pulmonary bacterial infection with $P<0.0167(0.05 / 3)$. 
Table 3. Complications and medication therapies by group.

\begin{tabular}{|c|c|c|c|c|}
\hline & $\begin{array}{c}\text { IPFI } \\
(n=106)\end{array}$ & $\begin{array}{l}\text { Pulmonary bacterial infection } \\
\qquad(\mathrm{n}=111)\end{array}$ & $\begin{array}{l}\text { Non-pulmonary infection } \\
\qquad(\mathrm{n}=106)\end{array}$ & $P$-value \\
\hline \multicolumn{5}{|l|}{ Complications } \\
\hline Agranulocytosis $\geqq 10$ days & $47(44.3)$ & $37(33.3)$ & $8(7.5)$ & $<0.001^{*+*}$ \\
\hline Hypoalbuminemia & $75(70.8)$ & $46(41.4)$ & $23(21.7)$ & $<0.001^{* * *}$ \\
\hline Electrolyte disorder & $81(76.4)$ & $57(51.4)$ & $28(26.4)$ & $<0.001^{* * *}$ \\
\hline Gastrointestinal fungal infection & $5(4.7)$ & $13(11.7)$ & $3(2.8)$ & $0.026^{*}$ \\
\hline Oral fungal infection & $10(9.4)$ & $2(1.8)$ & $1(0.9)$ & $0.006^{*}$ \\
\hline Urinary tract fungal infection & $2(1.9)$ & $1(0.9)$ & $0(0)$ & NA \\
\hline Epidermal fungal infection & $7(6.6)$ & $9(8.1)$ & $2(1.9)$ & 0.097 \\
\hline Systemic fungal infection & $1(0.9)$ & $0(0)$ & $0(0)$ & NA \\
\hline Sepsis & $13(12.3)$ & $6(5.4)$ & $0(0)$ & NA \\
\hline Drug induced hepatic damage & $15(14.2)$ & $10(9.0)$ & $5(4.7)$ & 0.060 \\
\hline Hyperuricemia & $8(7.5)$ & $8(7.2)$ & $15(14.2)$ & 0.155 \\
\hline \multicolumn{5}{|l|}{ Medication therapy } \\
\hline Venous Nutrition & $41(38.7)$ & $22(19.8)$ & $8(7.5)$ & $<0.001^{*+*}$ \\
\hline Albumin infusion & $33(31.1)$ & $12(10.8)$ & $3(2.8)$ & $<0.001^{*+*}$ \\
\hline Constructing central vein & $36(34.0)$ & $28(25.2)$ & $17(16.0)$ & $0.011^{* \ddagger}$ \\
\hline Use of glucocorticoid, days & & & & 0.075 \\
\hline 0 day & $49(46.2)$ & $65(58.6)$ & $52(49.1)$ & \\
\hline 1 to 5 days & $27(25.5)$ & $18(16.2)$ & $33(31.1)$ & \\
\hline$\geqq 6$ days & $30(28.3)$ & $28(25.2)$ & $21(19.8)$ & \\
\hline \multicolumn{5}{|c|}{ Number of different immunosuppressants } \\
\hline 0 & $15(14.2)$ & $6(5.4)$ & $7(6.6)$ & $0.005^{* \dagger}$ \\
\hline 1 to 3 & $76(71.7)$ & $101(91.0)$ & $91(85.8)$ & \\
\hline$\geqq 4$ & $15(14.2)$ & $4(3.6)$ & $8(7.5)$ & \\
\hline Number of antibiotics & & & & $<0.001^{* *}$ \\
\hline 0 & $5(4.7)$ & $1(0.9)$ & $80(75.5)$ & \\
\hline 1 to 3 & $65(61.3)$ & $75(67.6)$ & $22(20.8)$ & \\
\hline$\geqq 4$ & $36(34.0)$ & $35(31.5)$ & $4(3.8)$ & \\
\hline Days in antibiotics & & & & $<0.001^{* *}$ \\
\hline 0 days & $5(4.7)$ & $2(1.8)$ & $80(75.5)$ & \\
\hline 1 to 3 days & $10(9.4)$ & $4(3.6)$ & $2(1.9)$ & \\
\hline 4 to 7 days & $31(29.2)$ & $25(22.5)$ & $7(6.6)$ & \\
\hline$\geqq 8$ days & $60(56.6)$ & $80(72.1)$ & $17(16.0)$ & \\
\hline Days of Nystatin mouth rinse & & & & $<0.001^{* \dagger}$ \\
\hline 0 to 8 days & $93(87.7)$ & $55(49.5)$ & $84(79.2)$ & \\
\hline$\geqq 8$ days & $13(12.3)$ & $56(50.5)$ & $22(20.8)$ & \\
\hline
\end{tabular}

Data were summarized as $\mathrm{n}(\%)$ and compared among groups using Pearson Chi-square test or Fisher's exact test if cell numbers $<5$.

IPFI, invasive pulmonary fungal infections; NA, not assessed due to cell number of zero.

${ }^{\star} P<0.05$, indicated significantly different among groups.

$\dagger$ Indicates a significant difference between IPFI and Pulmonary bacterial infection with $P<0.0167(0.05 / 3)$.

$\ddagger$ Indicates a significant difference between IPFI and non-Pulmonary bacterial infection with $P<0.0167(0.05 / 3)$.

A total of 126 isolates were identified in 106 confirmed or clinical diagnosis cases of IPFI, divided between Candidas species (59.5\%) and mildew (40.5\%) (Table 5). The leading pathogen was Candida albicans, with Aspergillus species being the second most common fungus.

Step-wise multiple logistic regression analyses found that the risk factors associated with IPFI were previous history of IPFI, days of hospitalization, agranulocytosis lasting 10 or more days, hypoalbuminemia, and sepsis, while the number of days using Nystatin mouth rinse was a protective factor (Tables 6 and 7). The number of days of hospitalization was a strong risk factor in both analyses. The majority (95.28\%) of the IPFI patients were hospitalized more than 9 days, which was significantly longer than patients in either control group. The number of days of hospitalization was the leading risk factor for IPFI (OR: 37.752, 95\% CI: 8.737, 163.125) for patients with over a month of hospitalization when compared with patients without a pulmonary infection hospitalized less than 8 days. In the 
Table 4. IPFI prevalence rate in the years 2008 and 2009.

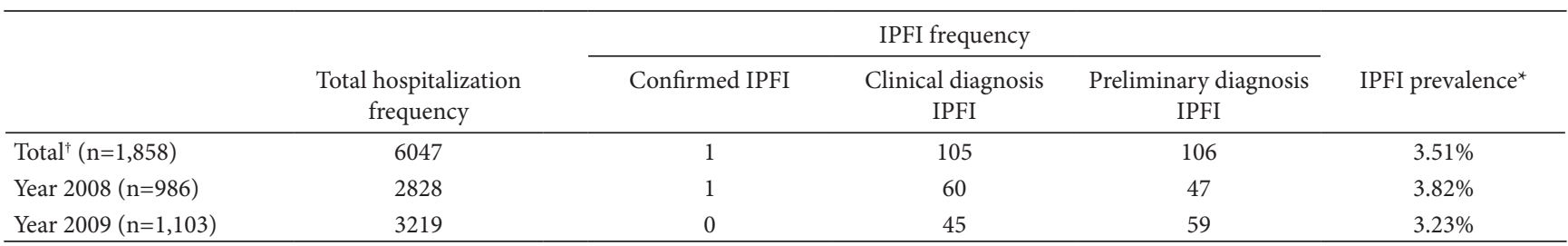

${ }^{*}$ IPFI prevalence $=$ Total IPFI frequency $/$ Total hospitalization frequency $\times 100 \%$

${ }^{\dagger}$ Some subjects had IPFI both in the year 2008 and in 2009.

comparison to patients with a bacterial pulmonary infection, the number of hospital days was the second most significant risk factor, after the risk from a previous case of IPFI.

Table 5. Summary of pathogens causing IPFI.

\begin{tabular}{lc}
\hline Pathogens & Frequency (\%) \\
\hline Total & $\mathbf{1 2 6}(\mathbf{1 0 0 \% )}$ \\
Candidas species & $\mathbf{7 5 ( 5 9 . 5 \% )}$ \\
$\quad$ Candidas albican & $64(50.7 \%)$ \\
Candidas glabrata & $3(2.4 \%)$ \\
Candidas tropicalis & $6(4.8 \%)$ \\
Candidas krusei & $1(0.8 \%)$ \\
Candidas guilliermondii & $1(0.8 \%)$ \\
Mildew species & $\mathbf{5 1}(\mathbf{4 0 . 5 \% )}$ \\
Aspergillus species & $47(37.3 \%)$ \\
Mucor species & $1(0.8 \%)$ \\
Penicillium species & $3(2.4 \%)$ \\
\hline
\end{tabular}

Table 6. Multivariate logistic regression analysis of risk factors ${ }^{\star}$ associated with IPFI relative to the pulmonary bacterial infection control group $(\mathrm{N}=217)$.

\begin{tabular}{lcc}
\hline Variables & OR $(95 \%$ CI. $)$ & $P$-value \\
\hline $\begin{array}{l}\text { Previous history of IPFI } \\
\text { Age, years }\end{array}$ & $6.402(1.678,24.427)$ & 0.007 \\
$\quad<60$ years & Reference & \\
$\quad \geqq 60$ years & $2.725(1.112,6.677)$ & 0.028 \\
$\begin{array}{l}\text { Days of hospitalization } \\
\quad<8 \text { days }\end{array}$ & Reference & \\
$\quad 9$ to 30 days & $5.649(1.601,19.933)$ & 0.007 \\
$\quad>30$ days & $37.752(8.737,163.125)$ & $<0.001$ \\
Complications & $3.524(1.475,8.419)$ & 0.005 \\
$\quad$ Agranulocytosis $\geqq 10$ days & $4.374(1.976,9.683)$ & $<0.001$ \\
$\quad$ hypoalbuminemia & $4.745(1.058,21.288)$ & 0.042 \\
$\quad$ Sepsis & Reference & \\
Days of Nystatin mouth rinse & $0.039(0.014,0.109)$ & $<0.001$ \\
$\quad 0$ to 8 days &
\end{tabular}

* Risk factors were chosen through step-wise multiple logistic regression analysis.

Results are shown as odds ratios (OR) with 95\% confidence intervals (95\% CI).
Agranulocytosis lasting more than 10 days increased the risk of IPFI, and it occurred in $44.3 \%$ of IPFI patients but only in $33.3 \%$ of patients with pulmonary bacterial infections and in only $7.5 \%$ of patients without pulmonary infections. Hypoalbuminemia developed in $70.8 \%$ of the patients in the IPFI group, and it increased the risk for IPFI by over fourfold. The accumulated number of antibiotics used to treat patients was also a risk factor. A total of $34 \%$ of patients with IPFI were treated with more than four antibiotics, while $75 \%$ of patients without pulmonary infections were treated with no antibiotics. Also, sepsis increased the risk of IPFI 4.5 -fold for patients with hematological malignancies who developed a pulmonary bacterial infection and subsequent sepsis. The risk of IPFI decreased with the use of Nystatin mouthwash. Underlying diseases did not affect the risk of IPFI.

Table 7. Multivariate logistic regression analysis of risk factors associated with IPFI relative to the non-pulmonary infection control group $(\mathrm{N}=212)$

\begin{tabular}{lcc}
\hline Variables & OR $(95 \%$ CI. $)$ & $P$-value \\
\hline $\begin{array}{l}\text { Previous history of IPFI } \\
\text { Age }\end{array}$ & $14.139(1.879,106.414)$ & 0.010 \\
$\quad<60$ years & Reference & \\
$\quad \geq 60$ years & $2.325(0.616,8.778)$ & 0.213 \\
Hospital days & Reference & \\
$\quad<8$ days & $24.687(4.534,134.418)$ & $<0.001$ \\
$\quad 9$ to 30 days & $546.497(36.069,8280.198)$ & $<0.001$ \\
$\quad>30$ days & & \\
& & \\
Complications & $5.363(1.072,26.825)$ & 0.041 \\
$\quad$ Agranulocytosis $\geqq 10$ days & $3.955(1.049,14.907)$ & 0.042 \\
$\quad$ Hypoalbuminemia & Reference & \\
The types of antibiotics & $46.146(8.824,241.32)$ & $<0.001$ \\
$\quad 0$ & $54.182(6.183,474.78)$ & $<0.001$ \\
1 to 3 & Reference & \\
$\quad \geqq 4$ & $0.006(0.001,0.049)$ & $<0.001$ \\
\hline Days of Nystatin mouth rinse & &
\end{tabular}

* Risk factors were chosen through step-wise multiple logistic regression analysis.

Results are shown as odds ratios (OR) with 95\% confidence intervals (95\% CI.). 


\section{Discussion}

The overall incidence of IPFI in our study was 3.5\%, in reasonable agreement with a recent report citing a rate from $3.7 \%$ to $8.8 \%$ [12] and slightly higher than a previous report of $2.9 \%$ [13]. The incidence of invasive fungal infections is increasing in recent years in patients with hematological malignancies [8,14-17]. An investigation of 1017 deceased patients with hematological malignancies found that $31 \%$ of them had IPFI, and $75 \%$ of those cases were not diagnosed as IPFI before death [15]. The incidence of IPFI among patients with hematological malignancies has been reported to be $4.6 \%$ to $18.1 \%[14,16,17]$. Among patients with hematological malignancies, IPFI causes the highest proportion of deaths and is a leading cause of their deaths [18].

Our study found that IPFI was caused by Candida albicans in $50.7 \%$ of the cases, and by Aspergillus species in $37.3 \%$, in reasonable agreement with previous reports [19]. The incidence of pulmonary Aspergilosis has been increasing since the 1990s [13-15,17,18].

The strong risk for IPFI was associated with prolonged hospitalization, in agreement with previous reports [20], indicates that the hospital is a unique ecological environment where patients frequently encounter pathogens. Longer hospital stays increase the chances of exposure to pathogens. Hematological malignancies and the techniques to diagnose and treat them, combined with increased pathogen exposure, compromise the defenses of patients and make them susceptible to infections in the hospital. This means that, as conditions permit, hospital stays should be as short as possible to reduce the incidence of hospital fungal infections. Also, when hospital stays are anticipated to be more than 9 days, measures should be taken to prevent IPFI.

Previous IPFI was a strong risk factor for IPFI in our study. In agreement, a comparison of IPFI patients and patients with pulmonary bacterial infections also found that previous IPFI was an independent risk factor for IPFI [21]. Patients with hematological malignancies have compromised immune function, so it is difficult to eradicate fungal infections with patient's own immune function. Immune function can be further compromised by chemotherapy, which can lead to a recurrence or dissemination of a fungal infection. This indicates that patients with a history of IPFI should be closely monitored clinically and that prevention of IPFI in these patients should be emphasized.

Agranulocytosis lasting 10 days or more increased the risk for IPFI in our study. An index of agranulocytosis, which monitors changes in the number of neutrophils over time, has been proposed to predict the risk of invasive mold infections [22]. Normal neutrophils are critical for non-specific immune responses and defend against fungal infections.

Concomitant hypoalbuminemia increased the risk for IPFI by over four-fold. A serum albumin level below $35 \mathrm{~g} / \mathrm{L}$ indicates protein-energy malnutrition. Malnutrition, particularly when severe, affects cellular immunity and humoral immunity, attenuates the ability of alveolar macrophages and neutrophils to kill pathogens, reduces the production of airway mucus, affects tissue repair, and increases the adhesion of pathogens to airway epithelial cells, which greatly increases susceptibility to fungal infections [23]. These concerns indicate the importance of nutritional support of patients with hematological malignancies and the importance of promptly rectifying hypoalbuminemia to prevent IPFI.

The accumulated number of antibiotics used to treat a patient is a risk factor for IPFI. Antibiotic treatments that are frequently altered may have a shorter effective concentration time or may not reach an effective concentration, which can lead to compromised clinical efficacy and possible flora imbalance that increases fungal infection susceptibility. These concerns make it necessary to emphasize the indications for treatment with antibiotics, avoid frequent altering of the antibiotics, detect the pathogens causing the infection, and detect the resistance state of the pathogens. These measures will help to prevent secondary fungal infection.

Our finding that bacterial sepsis increased the incidence of IPFI agrees with previous reports [24,25]. Sepsis indicates compromised immune function, and prophylactic anti-fungal treatment should be adopted while treating sepsis.

Though underlying diseases did not affect the risk of IPFI, their lack of effect may be due to the low proportion of patients with these diseases and to the major risks for IPFI coming from the causes mentioned above. The absence of glucocorticoid use as a risk factor may be because all the included patients had short-term glucocorticoid treatments.

This study is a retrospective single center research and the results gathered in this research may not apply to other institutes. The effect of Nystatin on preventing fungal infection needs to be examined in a case-controlled study in a larger group of patients.

In conclusion, we find the risk of IPFI in patients with hematological malignancies without HSCT include the days of hospitalization, history of IPFI, agranulocytosis lasting 10 or more days, hypoalbuminemia, the accumulated number of antibiotics, and sepsis. Awareness of these risk factors and taking actions to mitigate them can help to decrease the incidence of IPFI.

Acknowledgments: Kathy Boltz, PhD (Phoenix, AZ, USA) of MedCom Asia provided professional English-language writing assistance for this article. This study was supported by Fujian Medical University Youth Fund, \#2010MP020.

\section{References}

[1] SHELTON BK. Opportunistic fungal infections in the critically ill. Crit Care Nurs Clin North Am 2000; 12: 323-340

[2] HIGA M. Clinical epidemiology of fungal infection in diabetes. Jpn J Clin Med 2008; 66: 2239-2244 (in Japanese)

[3] JHAM BC, FRANÇA EC, OLIVEIRA RR, SANTOS VR, KOWALSKI LP et al. Candida oral et al.colonization and infection in Brazilian patients undergoing head and neck ra- 
diotherapy: a pilot study. Oral Surg Oral Med Oral Pathol Oral Radiol Endod 2007; 103: 355-358 http://dx.doi.org/10.1016/ j.tripleo.2006.02.005

[4] KONTOYIANNIS DP, MARR KA, PARKBJ, ALEXANDER BD, ANAISSIE EJ et al. Prospective surveillance for invasive fungal infections in hematopoietic stem cell transplant recipients, 2001-2006: overview of the Transplant-Associated Infection Surveillance Network (TRANSNET) Database. Clin Infect Dis 2010; 50: 1091-1100 http: //dx.doi.org/10.1086/651263

[5] NIVOIX Y, VELTEN M, LETSCHER-BRU V, MOGHADDAM A, NATARAJAN-AME $S$ et al. Factors associated with overall and attributable mortality in invasive aspergillosis. Clin Infect Dis 2008; 47: 1176-1184 http: //dx.doi. org/10.1086/592255

[6] OSTROSKY-ZEICHNER L, PAPPAS PG. Invasive candidiasis in the intensive care unit. Crit Care Med 2006; 34: 857-863 http: //dx.doi.org/10.1097/01.CCM.0000201897.78123.44

[7] VANDEWOUDE K, BLOT S, BENOIT D, DEPUYDT P, VOGELAERS D, COLARDYN F. Invasive aspergillosis in critically ill patients: analysis of risk factors for acquisition and mortality. Acta Clin Belg 2004; 59: 251-257 PMid: 15641394

[8] KOBAYASHI R, KANEDA M, SATO T, ICHIKAWA M, SUZUKI D, ARIGA T. The clinical feature of invasive fungal infection in pediatric patients with hematologic and malignant diseases: a 10-year analysis at a single institution at Japan. J Pediatr Hematol Oncol 2008; 30: 886-890 http: //dx.doi. org/10.1097/MPH.0b013e3181864a80

[9] OZYILMAZ E, AYDOGDU M, SUCAK G, AKI SZ, OZKURT $\mathrm{ZN}$ et al. Risk factors for fungal pulmonary infections in hematopoietic stem cell transplantation recipients: the role of iron overload. Bone Marrow Transplant 2010; 45: 1528-1533 http: //dx.doi.org/10.1038/bmt.2009.383

[10] WANG ZY, JIANG EL, ZHANG P, WANG H, BAO YS et al. Invasive fungal infections after allogeneic hematopoietic stem cell transplantation and related risk factors. J Exp Hematol 2008; 16: 618-622 (in Chinese)

[11] DENG WW. Standardizing the diagnosis and treatment of invasive pulmonary fungal diseases. Chin J Intern Med 2006;45: 623 (in Chinese)

[12] THEODORE S, LIAVA'A M, ANTIPPA P, WYNNE R, GRIGG A et al. Surgical management of invasive pulmonary fungal infection in hematology patients. Ann Thorac Surg 2009; 87: 1532-1538 http://dx.doi.org/10.1016/ j.athoracsur.2009.02.069

[13] SUN XF, HAN B, FENG J, ZHOU DB, WANG SJ et al. Clinical features of invasive pulmonary fungal infection secondary to malignant blood diseases. Acta Academiae Medicinae Sinicae 2009; 31: 575-579 (in Chinese)

[14] AUBERGER J, LASS-FLORL C, ULMER H, NOGLERSEMENITZ E, CLAUSEN J et al. Significant alterations in the epidemiology and treatment outcome of invasive fungal infections in patients with hematological malignancies. Int J Hematol 2008; 88: 508-515 http: //dx.doi.org/10.1007/s12185$\underline{008-0184-2}$
[15] CHAMILOS G, LUNA M, LEWIS RE, BODEY GP, CHEMALY $\mathrm{R}$ et al. Invasive fungal infections in patients with hematologic malignancies in a tertiary care cancer center: an autopsy study over a 15 -year period (1989-2003). Haematologica 2006; 91: 986-989

[16] HAHN-AST C, GLASMACHER A, MUCKTER S, SCHMITZ A, KRAEMER A et al. Overall survival and fungal infectionrelated mortality in patients with invasive fungal infection and neutropenia after myelosuppressive chemotherapy in a tertiary care centre from 1995 to 2006. J Antimicrob Chemother 2010; 65: 761-768 http: //dx.doi.org/10.1093/ jac/dkp507

[17] PAGANO L, CAIRA M, CANDONI A, OFFIDANI M, FIANCHI $L$ et al. The epidemiology of fungal infections in patients with hematologic malignancies: the SEIFEM-2004 study. Haematologica 2006; 91: 1068-1075

[18] SHEN ZX. Diagnostic criteria and therapeutic principle of invasive fungal infection in hematological diseases or malignant tumours. Chin J Intern Med 2007;46: 532-533 (in Chinese)

[19] HOENIGL M, ZOLLNER-SCHWETZ I, SILL H, LINKESCH W, LASS-FLORL C et al. Epidemiology of invasive fungal infections and rationale for antifungal therapy in patients with haematological malignancies. Mycoses 2011; 54: 454-459 http: //dx.doi.org/10.1111/j.1439-0507.2010.01881.x

[20] CORNELY OA, AVERSA F, COOK P, JONES B, MICHALLET $\mathrm{M}$ et al. Evaluating the role of prophylaxis in the management of invasive fungal infections in patients with hematologic malignancy. Eur J Haematol 2011; 87: 289-301 http: //dx.doi. org/10.1111/j.1600-0609.2011.01682.x

[21] TAN XJ, MENG FY, QIN JZ, LIU LX. Analysis of the risk factors of pulmonary fungal infections related to hematologic malignancies. J South Med Univ 2009;29: 2506-2509 (in Chinese)

[22] PORTUGAL RD, GARNICA M, NUCCI M. Index to predict invasive mold infection in high-risk neutropenic patients based on the area over the neutrophil curve. J Clin Oncol 2009;27: 3849-3854 http: //dx.doi.org/10.1200/ LCO.2008.21.0856

[23] SCHOLS AM, CREUTZBERG EC, BUURMAN WA, CAMPFIELD LA, SARIS WH et al. Plasma leptin is related to proinflammatory status and dietary intake in patients with chronic obstructive pulmonary disease. Am J Respir Crit Care Med 1999; 160: 1220-1226.

[24] BOW EJ, LOEWEN R, CHEANG MS, SCHACTER B. Invasive fungal disease in adults undergoing remission-induction therapy for acute myeloid leukemia: the pathogenetic role of the antileukemic regimen. Clin Infect Dis 1995; 21: 361-369 http: //dx.doi.org/10.1093/clinids/21.2.361

[25] SPARRELID E, HAGGLUND H, REMBERGER M, RINGDEN O, LONNQVIST B et al. Bacteraemia during the aplastic phase after allogeneic bone marrow transplantation is associated with early death from invasive fungal infection. Bone Marrow Transplant 1998; 22: 795-800 http: //dx.doi. org/10.1038/sj.bmt.1701404 